\title{
Topological router induced via long-range hopping in a Su-Schrieffer-Heeger chain
}

\author{
Lu Qi, ${ }^{1}$ Yu Yan, ${ }^{1}$ Yan Xing, ${ }^{1}$ Xue-Dong Zhao, ${ }^{1}$ Shutian Liu, ${ }^{1,}$ Wen-Xue Cui, ${ }^{2}$ Xue Han, ${ }^{2}$ \\ Shou Zhang, ${ }^{2, \dagger}$ and Hong-Fu Wang (1) ${ }^{2, \dagger}$ \\ ${ }^{1}$ School of Physics, Harbin Institute of Technology, Harbin, Heilongjiang 150001, China \\ ${ }^{2}$ Department of Physics, College of Science, Yanbian University, Yanji, Jilin 133002, China
}

(Received 25 November 2020; accepted 25 March 2021; published 12 April 2021)

\begin{abstract}
We show how to implement the various kinds of topological routers via introducing the specific long-range hopping in the one-dimensional modulated Su-Schrieffer-Heeger chain. We find that the long-range hopping with hopping amplitudes identical to those of the intercell hopping between the first site and other odd sites holds a distinctive topological channel (gap state), by which the particle initially prepared at the last site can appear at the first site and all of the even sites with approximately equal probabilities. This extraordinary performance indicates that, from the perspective of treating the last site as the input port and treating the first site and other even sites as output ports, the present system can be naturally equivalent to a distribution device with multiple output ports, i.e., the topological router. We show that the number of the output ports for the present topological router can be flexibly tuned via reducing the long-range hopping terms. Especially, the system experiences a phase transition with the increasing of the long-range hopping amplitudes, in which the original gap state becomes a topological channel to implement the topological router with the output ports only at all even sites. We stress that both the two kinds of topological routers are protected by the energy gap and hence are robust to the mild disorder added into the system. Moreover, together with the construction of the topological interface, we find that the topological routers can own the output ports in a symmetrical way about the interface site. The topological routers may play the pivotal role in the large-scale entanglement distributions, which significantly expands and supplies the potential applications of the topological materials in quantum information processing.
\end{abstract}

DOI: 10.1103/PhysRevResearch.3.023037

\section{INTRODUCTION}

Quantum information processing [1-4] has become one of the most concerning research hot spots in recent years. Different from the classical information processing, quantum information processing is a brand-new way of information processing since the carrier of information in quantum information processing is quantum states [5-11] rather than the classical bits, leading it to own the unique advantages in achieving higher information processing efficiency and providing more storage space of information. Especially, in the large-scale quantum information processing, the robust quantum state transfer [12-16] is one of the key elements to realize quantum information transmission between remote nodes. In this way, it is necessary to construct an effective quantum state transmission channel based on various quantum systems, including the quantum dots [17-20], cavity quantum electrodynamics [21-24], and nitrogen-vacancy centers [25-27].

\footnotetext{
*stliu@hit.edu.cn

†szhang@ybu.edu.cn

‡hfwang@ybu.edu.cn
}

Published by the American Physical Society under the terms of the Creative Commons Attribution 4.0 International license. Further distribution of this work must maintain attribution to the author(s) and the published article's title, journal citation, and DOI.
However, these systems inevitably possess the perturbation and decoherence process due to the existence of the artificial variance or the spontaneous defects of the systems, which may greatly reduce the reliability of the quantum information transmission. Thus, how to implement the robust channel of the quantum state transfer is still an urgent and continuously followed issue.

Topological insulators [28-31], as one of the new classifications for materials, has attracted much more attention due to the manifold novel characteristics, such as the simultaneous existences of the insulating bulk and conducting edge states. These conducting edge states are protected by the energy gap and hence are immune to the mild disorder and perturbation [32-36] added into the system. This prominent performance leads the topological edge states to become the promising candidate to implement the robust topological quantum state transfer [37-41]. The central concept for the quantum state transfer based on the topological edge channel can be comprehended via the topological edge pumping. Note that the topological edge pumping has been proposed and observed both in the periodic [42] and quasiperiodic quasicrystal [43] systems. The robust quantum state transfer via topologically protected edge pumping in two-dimensional topological spin systems has also been reported in Ref. [44]. Subsequently, the robust quantum state transfer scheme assisted by the topological edge states has been realized based on the one-dimensional superconducting qubit chains [45], in which the initial state prepared at the first site can be transferred into the last site via 
the adiabatic edge pumping. It is worth noting that the limitation of the adiabatic condition leads the process of the state transfer inevitably to consume many more time resources. To handle this obstacle, the accelerated quantum state transfers by dint of the topological edge channels assisted by adiabatic passage [46] and long-range hopping [47] have been proposed recently. Based on the concept of the topological state transfer, in Ref. [48], we demonstrate that the gap state induced by the on-site energy and next-nearest-neighbor (NNN) hopping can be used as the topological channel to implement the special topological quantum state transfer, in which the initial state prepared at the right edge can be transferred into the first two sites with approximately equal probabilities. The scheme mentioned in Ref. [48] actually indicates the potential feasibility of distributing the initial state between several nodes, which is expected to provide the prototype to implement the large-scale topological quantum information processing. Unfortunately, the scheme mentioned above only owns two output ports, which obviously is insufficient for the expected large-scale topological quantum information processing. Thus, how to construct a topological channel with multiple output ports to implement the quantum state transfer is still an open problem.

In this paper, inspired by the motivation mentioned above, we try to induce one specific topological channel via introducing the elaborate long-range hopping in the modulated Su-Schrieffer-Heeger (SSH) model with the odd number of lattice sites. We demonstrate that the long-range hopping with the same strength as the intercell hopping added on the first site and all of the other odd sites indeed induces a special gap state, in which the gap state has the uniform distributions at the first site and all of the even sites when the modulated parameter in a certain range. We find that, based on this special topological channel, the initial state prepared only at the last site can be transferred into the first site and all of the even sites with the approximately equal probabilities. This special topological state transfer, if we treat the last site as the input port and treat the first and all of even sites as the output ports, can be naturally equivalent to a router in form. The present router is spontaneously immune to the mild disorder due to the topological protection originating from the energy gap. We reveal that the number of the output ports can be tuned via reducing the long-range hopping terms. Significantly, we find that the system experiences a phase transition with the increasing of long-range hopping amplitudes, after which, the output ports of the original topological router are only occupied on all of the even sites. Furthermore, we also show the topological routers assisted by the topological interface, in which the initial particle can realize the symmetrical splittings throughout the topological edge pumping. We stress that the present several topological routers can be realized under the current experimental conditions, which greatly expands the possible applications of the topological matter in quantum information processing, such as the large-scale quantum distributions.

The paper is organized as follows: In Sec. II, we demonstrate that the topological router can be induced via the long-range hopping added between the first site and all of other odd sites. We reveal that the different kinds of topological router can be induced by the weak long-range hopping, the large long-range hopping, and the topological interface.

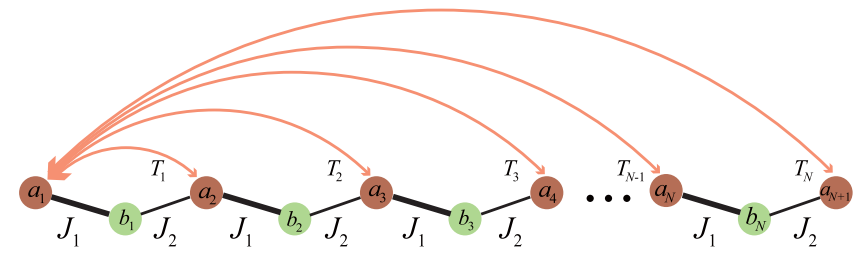

FIG. 1. The diagrammatic sketch of the modulated SuSchrieffer-Heeger (SSH) model with the long-range hopping. The size of the SSH chain is $L=2 N+1$ with $N$ unit cells, in which each unit cell contains two sublattice sites $a_{n}$ and $b_{n}$. The intraand intercell coupling between two adjacent sites is $J_{1}$ and $J_{2}$. Note that the SSH chain has the special long-range hopping between the first site $a_{1}$ and all other $a$-type sites $a_{n=2, \ldots, N+1}$ with the amplitude $T_{n=1, \ldots, N}$.

In Sec. III, we show that the topological router can be implemented under the current experimental conditions. Finally, a conclusion is given in Sec. IV.

\section{TOPOLOGICAL ROUTER INDUCED BY LONG-RANGE HOPPING}

Consider a 1D SSH chain with the size of $L=2 N+1$ ( $N$ is the number of unit cells and we take $N$ as even in the following), as shown in Fig. 1. In this chain, each unit cell contains two sublattice sites $a_{n}$ and $b_{n}$ with the intra- and intercell coupling $J_{1}$ and $J_{2}$. Especially, the SSH chain owns the elaborate long-range hopping, in which the first $a$-type site $a_{1}$ can support the long-range hopping with an arbitrary $a$-type site in the $n$th $(n=2,3, \ldots, N+1)$ unit cell accompanied with the hopping amplitude $T_{n}(n=1,2, \ldots, N)$. The SSH chain mentioned above can be described by the following Hamiltonian, with

$$
H=\sum_{n=1}^{N}\left[\left(J_{1} a_{n}^{\dagger} b_{n}+J_{2} a_{n+1}^{\dagger} b_{n}+T_{n} a_{1}^{\dagger} a_{n+1}\right)+\text { H.c. }\right],
$$

where $J_{1}=J+\cos \theta$ and $J_{2}=J-\cos \theta$ are the modulated nearest-neighbor $(\mathrm{NN})$ hopping amplitudes with $\theta \in[0,2 \pi]$ being the periodic parameter. For simplicity, here, we choose $J=1$ as the energy unit in all of the paper. Note that, when the long-range hopping terms in Eq. (1) are vanishing $\left(T_{n}=0\right)$, the present SSH chain becomes a standard SSH model with modulation, in which the system possesses a zero-energy topological right (left) edge state within $\theta \in$ $[0,0.5 \pi] \cup[1.5 \pi, 2 \pi](\theta \in[0.5 \pi, 1.5 \pi])$. The zero-energy topological edge states in modulated SSH chain are widely used as the topological channel to implement the robust quantum state transfer [37-40]. The question is whether the channel of quantum state transfer can still hold when the longrange hopping between $a$-type sites is added into the system. In the following, we will explore the effects of the long-range hopping between $a$-type sites on the topological channel.

\section{A. Topological router induced by weak long-range hopping}

For simplicity, we choose the long-range hopping amplitudes to take $T_{1}=T_{2}=\ldots=T_{N}=J_{2}$. The energy spectrum, when the long-range hopping is added into the system, is 

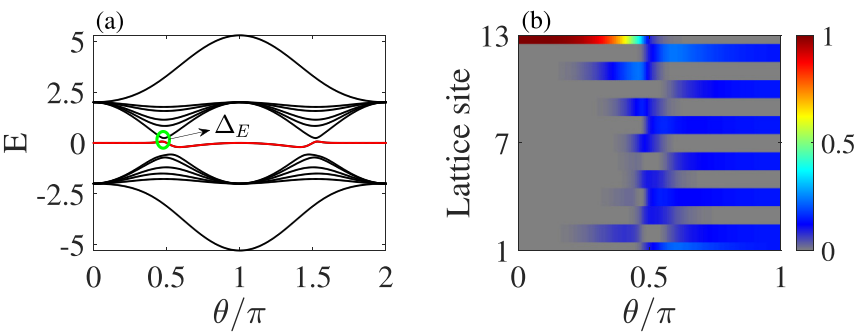

(c)

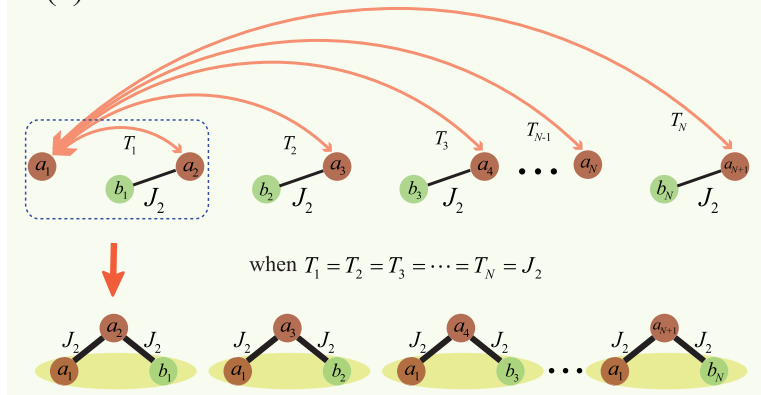

FIG. 2. Energy spectrum, distribution of gap state, and diagrammatic sketch of the localized state. (a) The energy spectrum of the SSH chain when $T_{1}=T_{2}=\ldots=T_{N}=J_{2}$. The energy spectrum has a gap state (red line). The minimal energy space between the gap state and the up energy band is labeled as $\Delta_{E}$. (b) The distribution of the gap state versus the periodic parameter $\theta$, in which the gap state is mainly localized at the last site within $\theta \in[0,0.5 \pi]$ while it is mainly localized at sites $a_{1}, b_{1}, b_{2}, \ldots$, and $b_{N}$ uniformly within $\theta \in[0.5 \pi, \pi]$. Note that the distribution of the gap state when $\theta \in[\pi, 1.5 \pi](\theta \in[1.5 \pi, 2 \pi])$ has the same form as the distribution in $\theta \in[0.5 \pi, \pi](\theta \in[0,0.5 \pi])$. (c) The diagrammatic sketch of the distribution for the gap state when $\theta \in[0.5 \pi, \pi]$. When $\theta \in$ $[0.5 \pi, \pi]$, the intra- and intercell coupling satisfy $J_{1}<J_{2}$, implying the weak bond $J_{1}$ and strong bond $J_{2}$. Under the limiting of the weak bond, the SSH chain is divided into several components in the unit of three sites, such as $a_{1}, a_{2}\left(a_{3}, \ldots, a_{N+1}\right)$, and $b_{1}\left(b_{2}, \ldots, b_{N}\right)$. When the long-range hopping satisfies $T_{n=1, \ldots, N}=J_{2}$, the strong bonds $T_{n}$ and $J_{2}$ generate a domain wall (see Fig. 1.8 in Ref. [49]), leading the sites $a_{1}$ and $b_{1}\left(b_{2}, \ldots, b_{N}\right)$ to be isolated. These isolated sites just correspond to the distribution of the gap state within $\theta \in[0.5 \pi, \pi]$. The other parameter takes $L=2 N+1$ with $N=6$. The unit is $J=1$.

depicted in Fig. 2(a). Similar to the case of vanishing longrange hopping, the present energy spectrum also possesses a gap state in the whole energy gap. However, the difference is that the gap state does not keep the zero energy due to the introduction of long-range hopping. Especially, the minimal energy space $\Delta_{E}$ between the gap state and the up band becomes much narrower since the long-range hopping is only added on the $a$-type sites. The distribution of the gap state is further plotted in Fig. 2(b), in which the gap state is mainly localized at the last site $a_{N+1}$ within $\theta \in[0,0.5 \pi]$ while it is uniformly distributed at sites $a_{1}, b_{1}, b_{2}, \ldots$, and $b_{N}$ within $\theta \in[0.5 \pi, \pi]$. The reason is that, when $\theta \in[0,0.5 \pi]$, the $\mathrm{NN}$ and long-range hopping amplitudes satisfy $J_{1}>J_{2}=T_{n}$, indicating that the two sublattice sites belonging to one unit cell are paired due to the existence of the strong bond $J_{1}$. The paired two sublattice sites further lead the last site $a_{N+1}$ to be isolated and become the topological right edge state. On the contrary, when $\theta \in[0.5 \pi, \pi]$, the $\mathrm{NN}$ and long-range hopping amplitudes now become $J_{1}<J_{2}=T_{n}$. As shown in Fig. 2(c), the weak bond $J_{1}$ and two strong bonds $J_{2}=T_{n}$ lead that the three sites, such as $a_{1}, a_{2}\left(a_{3}, a_{4}, \ldots, a_{N+1}\right)$, and $b_{1}\left(b_{2}, b_{3}\right.$, $\left.\ldots, b_{N}\right)$ generate a domain wall [49], which further induces the two sites $a_{1}$ and $b_{1}\left(b_{2, \ldots, N}\right)$ to be isolated. As a result, the gap state is mainly localized at sites $a_{1}, b_{1}, b_{2}, \ldots$, and $b_{N}$ uniformly when $\theta \in[0.5 \pi, \pi]$.

This special distribution of the gap state induced by the long-range hopping $T_{n}$ indicates that, if we prepare the particle at last site $a_{N+1}$ initially, with the varying of parameter $\theta$ from 0 to $\pi$, the initial particle will be finally transferred into the sites $a_{1}, b_{1}, b_{2}, \ldots$, and $b_{N}$ uniformly. This transfer process actually implies that the gap state can be used as the topological channel to implement the state transfer between the initial state $|\Psi\rangle_{i, 1}=|0,0,0,0, \ldots, 0,0,1\rangle$ and the final state $|\Psi\rangle_{f, 1}=\frac{1}{\sqrt{N+1}}|1,1,0,1, \ldots, 0,1,0\rangle$. Note that here we define the states in the particle number space, in which the probability of the particle at each site obeys the statistics of a single particle.

To implement the topological state transfer mentioned above, we should rewrite the periodic parameter $\theta$ as the time-dependent version $\theta=\Omega t$ with the ramping speed $\Omega$ and time $t$. In this way, the initial state $|\Psi\rangle_{i, 1}$ will be evolved along the gap state under the domination of $i \frac{\partial}{\partial_{t}}|\Psi\rangle_{i, 1}=$ $H\left(\theta_{t}\right)|\Psi\rangle_{i, 1}$. Note that, due to the topological protection of the minimal energy space $\Delta_{E}$, the evolution process is naturally immune to the mild perturbation, e.g., the on-site disorder $\sum_{n} W\left(\delta_{a, n} a_{n}^{\dagger} a_{n}+\delta_{b, n} b_{n}^{\dagger} b_{n}\right)$ (with $W$ being the disorder strength and $\delta_{a, n}\left(\delta_{b, n}\right)$ being a random number within $[-0.5,0.5])$. We plot the fidelity between the evolved final state and the ideal final state $|\Psi\rangle_{f, 1}$ versus the ramping speed $\Omega$ and the disorder strength $W$, as shown in Fig. 3(a). The numerical results clearly show that, for the slow enough ramping speed $\log _{10}(\Omega)<-2.5 J$ and mild enough disorder strength $W<0.5 J$, the state transfer between $|\Psi\rangle_{i, 1}$ and $|\Psi\rangle_{f, 1}$ can be realized with a high enough fidelity. We stress that the former condition of $\log _{10}(\Omega)<-2.5 \mathrm{~J}$ originates from the adiabatic evolution demand, while the latter condition of $W<0.5 \mathrm{~J}$ is associated with the topological protection originating from the energy gap. We also depict the state transfer process when $\Omega=5 \times 10^{-4} \mathrm{~J}$ and $W=0.25 \mathrm{~J}$, as shown in Fig. 3(b). Obviously, when the mild on-site disorder is added into the system, the state transfer between $|\Psi\rangle_{i, 1}$ and $|\Psi\rangle_{f, 1}$ can indeed be implemented. Furthermore, we also investigate the state transfer process when the disorder is added into the NN hopping, as shown in Figs. 3(c) and 3(d). The numerical results reveal conclusions similar to the cases shown in Figs. 3(a) and 3(b).

These results indicate that, if we regard the last site $a_{N+1}$ as the input port and regard the sites $a_{1}, b_{1}, b_{2}, \ldots$, and $b_{N}$ as multiple output ports, the state transfer process mentioned above is actually equivalent to a router in function [see Fig. 3(e)], in which the particle initially prepared at the input port can be distributed toward multiple output ports. Especially, since the distribution process is assisted by the topological gap state, the present state transfer is thus equivalent to a topological router and naturally immune to the mild 

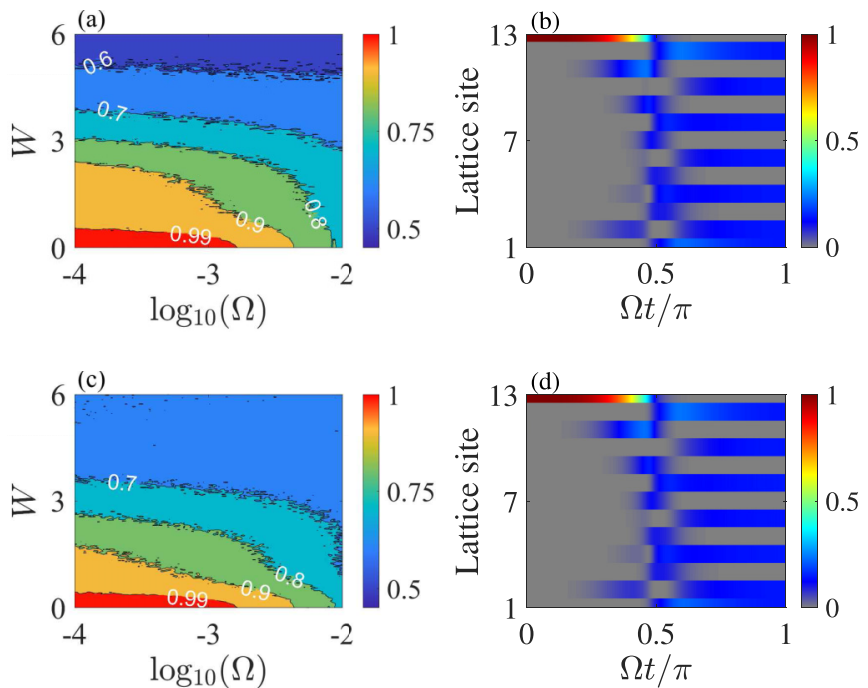

\section{(e)}

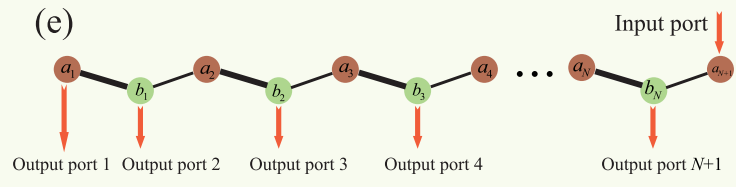

(ons

analogous to

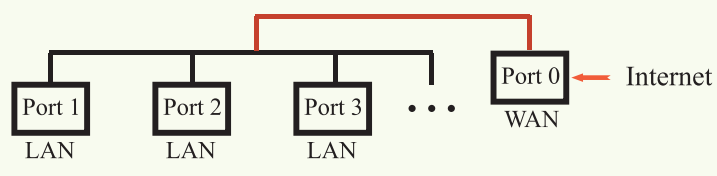

FIG. 3. Fidelity, evolution process of the initial state, and diagrammatic sketch of the topological router. (a) The fidelity between the evolved final state and the ideal final state $|\Psi\rangle_{f, 1}$ versus the ramping speed $\Omega$ and on-site disorder strength $W$. Note that here we only focus on the process of the state transfer and hence artificially abandon the phase information of the evolved state in the process of calculating the fidelity. The disorder is randomized 100 times and the average is taken. (b) The process of the evolution for the initial state $|\Psi\rangle_{i, 1}$ when the on-site disorder is added into the system, in which the parameters satisfy $\Omega=5 \times 10^{-4} \mathrm{~J}$ and $W=0.25 \mathrm{~J}$. (c) The fidelity between the evolved final state and the ideal final state $|\Psi\rangle_{f, 1}$ versus the ramping speed $\Omega$ and NN disorder strength $W$. (d) The process of the evolution for the initial state $|\Psi\rangle_{i, 1}$ when the NN disorder is added into the system with $\Omega=5 \times 10^{-4} \mathrm{~J}$ and $W=0.25 \mathrm{~J}$. (e) If we treat the last site $a_{N+1}$ as the input port and treat the sites $a_{1}, b_{1}$, $b_{2}, \ldots$, and $b_{N}$ as output ports, the present process of the state transfer is naturally equivalent to a router in form. The other parameter takes $L=2 N+1$ with $N=6$. The unit is $J=1$.

local perturbation. The robustness of the topological router against the local perturbation greatly decreases the obstacle in practical applications. Moreover, we stress that the number of the output ports in the topological router can be flexibly tuned via reducing the terms of the long-range hopping $T_{n}$; e.g., when $T_{1}=T_{2}=\ldots=T_{N-1}=J_{2}$, the output ports of the topological router will be reduced by 1 (see Appendix for more discussions). In this way, we can tune the number of the output ports via reducing or adding the long-range hopping terms between $a$-type sites.

Actually, the topological router is a kind of special topological pumping in the SSH model, in which the particle initially prepared at the input port can be pumped toward several output ports. Compared with the traditional topological edge pumping in the SSH model [45-47], the dominant advantage of the present topological router is that the topological router can realize the topological distributions at several output ports, in which the number of the output ports can be flexibly tuned via designing the long-range hopping terms. More specifically, this topological pumping mentioned in Refs. [45-47] can only realize the pumping of the particle from one edge to another edge. In other words, these pumping schemes, especially in Refs. [46,47], are much more focused on the transfer efficiency than the scalability (large-scale distribution). In the quantum network, efficiency and scalability are the basis of the high-efficiency and large-scale quantum information processing. The topological edge pumping via topological channel actually plays the role in removing the obstacles of the fast and robust excitation transmission, which has been explored extensively and maturely. However, the investigations about the scalability or the large-scale distribution of the quantum network by dint of the topological channel are still relatively scarce so far. The concept of the topological router based on the topological edge channel, as far as we know, seems not to be proposed yet. The topological router actually deals with the explorations of the large-scale distribution in the quantum network based on topological matters. In addition to the traditional topological edge pumping in the SSH model, note that the two edge states will experience the Rabi-type flopping for the chain with the even size of lattice [38], by which the topological beam splitter with the tunable output proportions at two output ports can be constructed [41]. The similar topological beam splitter also has been proposed in Ref. [48] via introducing the NNN hopping. Compared with the traditional topological edge pumping in the SSH model, these topological beam splitter schemes possess two output ports, which takes the first step toward the large-scale topological distributions. However, these topological beam splitters only have two output ports, which is obviously insufficient for the expected large-scale quantum information processing. Thus, the appearance of the topological router with multiple output ports will greatly supply the applications of the topological matters in large-scale quantum information processing. Especially, the tunable number of the output ports via modulating the long-range hopping terms makes the practical application of the topological router in a quantum network to be much more universal and flexible.

\section{B. Topological router induced by strong long-range hopping}

In Sec. II A, we have demonstrated and shown that the long-range hopping amplitudes $T_{1}=T_{2}=\ldots=T_{N}=J_{2}$ can induce a topological router. Here, we will investigate the effects of the increasing long-range hopping amplitudes $T_{1}=$ $T_{2}=\ldots=T_{N}=T J_{2}$ with $T>1$ on the topological router. We stress that the topological router is protected by the energy gap, and thus is closely related to the minimal energy space $\Delta_{E}$ between the gap state and the up energy bands. The vary- 

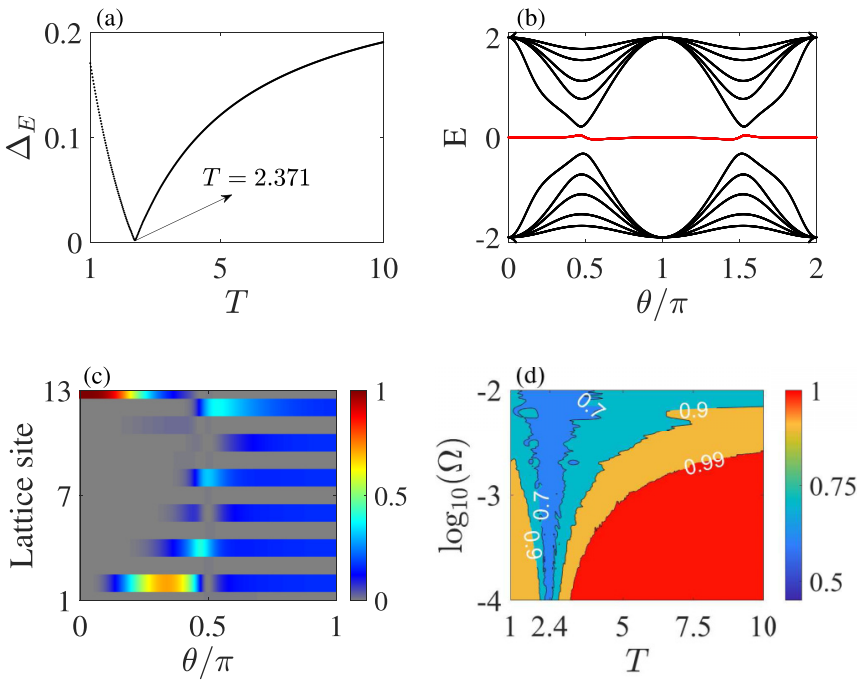

(e)

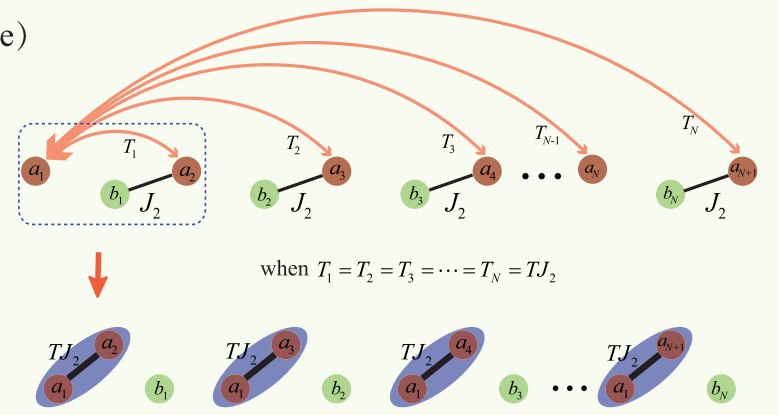

FIG. 4. (a) The varying of minimal energy space $\Delta_{E}$ with the increasing of the long-range hopping amplitude. (b) The energy spectrum around the gap under the large long-range hopping amplitude condition with $T=8 \mathrm{~J}$. The energy spectrum still has a gap state. (c) The distribution of the gap state. (d) The fidelity between the evolved final state and the state $|\Psi\rangle_{f, 1}^{\prime}$ versus the ramping speed $\Omega$ and $T$. The fidelity exhibits a clear dividing line around $T=2.4 \mathrm{~J}$. (e) The diagrammatic sketch of the distribution for the gap state when $\theta \in[0.5 \pi, \pi]$. Compared with the case shown in Fig. 2(e), the strong enough long-range hopping amplitudes $T_{n=1, \ldots, N}=T J_{2}$ lead the two sites $a_{1}$ and $a_{2}\left(a_{3}, \ldots, a_{N+1}\right)$ to be paired, further leading the sites $b_{1}, b_{2}, \ldots, b_{N}$ to be isolated. The other parameter takes $L=2 N+1$ with $N=6$. The unit is $J=1$.

ing of the minimal energy space $\Delta_{E}$ versus the parameter $T$ is plotted in Fig. 4(a). We find that the minimal energy space $\Delta_{E}$ decreases first and increases subsequently with the increasing of the parameter $T$. Especially, the minimal energy space $\Delta_{E}$ closely reaches to $\Delta_{E} \approx 0$ when $T \approx 2.371 J$, implying that the system may experience a phase transition.

To explore the possible phase transition induced by the large parameter $T$, we plot the energy spectrum and the distribution of the gap state when $T=8 \mathrm{~J}$, as shown in Figs. 4(b) and 4(c). The energy spectrum still holds a gap state and the gap state is uniformly localized at the sites $b_{1}$, $b_{2}, \ldots$, and $b_{N}$ when $\theta \in[0.5 \pi, \pi]$. The distribution of the new gap state induced by large $T$ indicates the feasibility of implementing the state transfer between the initial state $|\Psi\rangle_{i, 1}=|0,0,0,0, \ldots, 0,0,1\rangle$ and the final state $|\Psi\rangle_{f, 1}^{\prime}=$ $\frac{1}{\sqrt{N}}|0,1,0,1, \ldots, 0,1,0\rangle$. To further estimate the feasibility of implementing the state transfer between $|\Psi\rangle_{i, 1}$ and $|\Psi\rangle_{f, 1}^{\prime}$, we plot the fidelity of the state transfer versus the ramping speed $\Omega$ and the parameter $T$, as shown in Fig. 4(d). The numerical results reveal that, corresponding to the small enough ramping speed $\log _{10}(\Omega)<10^{-3} J$, the large enough parameter $T$ with $T>2.4 J$ ensures that the state transfer between $|\Psi\rangle_{i, 1}$ and $|\Psi\rangle_{f, 1}^{\prime}$ can be realized with a high enough fidelity.

The new state transfer between $|\Psi\rangle_{i, 1}$ and $|\Psi\rangle_{f, 1}^{\prime}$ actually corresponds a new topological router, in which the particle initially injected into the input port $a_{N+1}$ is finally divided into $N$ parts appearing at all of $b$-type site ports. Note that, as depicted in Fig. 4(a), the minimal energy space $\Delta_{E}$ gradually increases with $T$ increasing from $T \approx 2.371 J$. The enlarged minimal energy space $\Delta_{E}$ means that the topological protection of the new topological router is strengthened. Dramatically, when the parameter $T$ satisfies $T>7.8 \mathrm{~J}$, the minimal energy space $\Delta_{E}$ of the new topological router becomes larger than the topological router mentioned in Sec. II A, implying that the new topological router is much more robust against the local perturbations.

The new topological router can be further comprehended via the diagrammatic sketch shown in Fig. 4(e). Compared with the case in Fig. 2(c), when $\theta \in[0.5 \pi, \pi]$, the large enough $T$ with $T \gg 1$ makes the two sites $a_{1}$ and $a_{2}$ $\left(a_{3}, a_{4}, \ldots, a_{N+1}\right)$ to be paired due to the strong enough bonds $T_{n}=T J_{2}$, leading to the sites $b_{1}, b_{2}, \ldots$, and $b_{N}$ being isolated. These isolated sites $b_{1}, b_{2}, \ldots$, and $b_{N}$ just correspond to the output ports of the new topological router. The appearance of the new topological router indicates that, in addition to the tunable ports induced by reducing the long-range hopping terms, we also can induce two different kinds of topological routers via controlling the strength of the long-range hopping. Thus, via designing the long-range hopping terms reasonably, we can realize various topological routers depending on the different target, which greatly expands the potential applications of topological materials in quantum information processing.

\section{Topological router induced by topological interface}

Now, we still consider a SSH chain with the size of $L=$ $2 N+1$ ( $N \in$ even), but the intra- and intercell NN coupling configurations have a rotation after the $a$-type site in the $(N / 2+1)$ th unit cell, as shown in Fig. 5. Naturally, the SSH chain generates an interface at the site $a_{N / 2+1}$. Note that the restriction condition of even $N$ ensures that the interface is located at the $a$-type site. Similarly to the case discussed in Fig. 1, we also symmetrically add the long-range hopping between the two sides of the interface site, as shown in Fig. 5. In this way, the present SSH chain is equivalent to the same (mirror-symmetric) version as the model shown in Fig. 1 when $n \in\left[1,2, \ldots, \frac{N}{2}\right]\left(n \in\left[\frac{N}{2}+1, \frac{N}{2}+2, \ldots, N\right]\right)$. The Hamiltonian of the present SSH chain can be written as

$$
\begin{aligned}
H= & \sum_{n=1}^{N / 2}\left[\left(J_{1} a_{n}^{\dagger} b_{n}+J_{2} a_{n+1}^{\dagger} b_{n}+T_{n} a_{1}^{\dagger} a_{n+1}\right)+\text { H.c. }\right] \\
& +\sum_{n=N / 2+1}^{N}\left[\left(J_{2} a_{n}^{\dagger} b_{n}+J_{1} a_{n+1}^{\dagger} b_{n}+T_{n} a_{N+1}^{\dagger} a_{n}\right)+\text { H.c. }\right],
\end{aligned}
$$




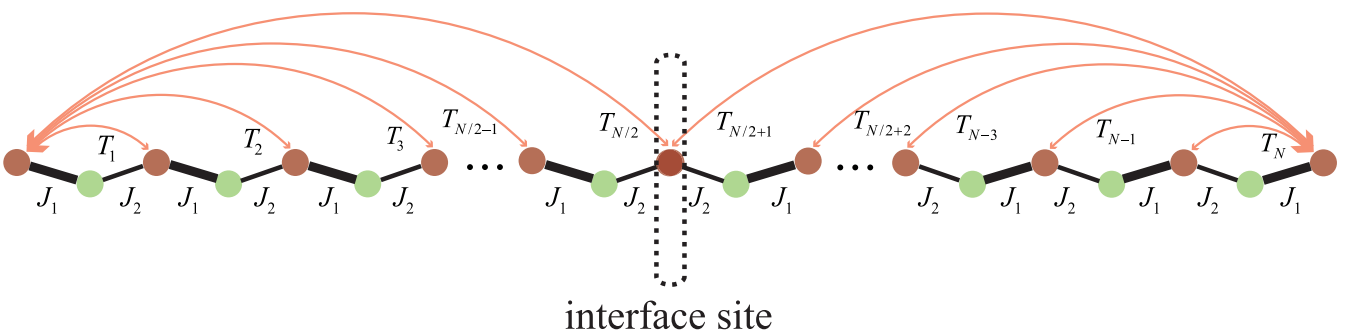

FIG. 5. The diagrammatic sketch of the modulated Su-Schrieffer-Heeger (SSH) model with the interface. The size of the SSH chain is $L=2 N+1$ with $N$ unit cells $\left(N \in\right.$ even). The SSH chain has the intra- and intercell coupling $J_{1}\left(J_{2}\right)$ and $J_{2}\left(J_{1}\right)$ before (after) the interface site $a_{N / 2+1}$. Note that the SSH chain has the long-range hopping between the first site $a_{1}$ and all other $a$-type sites $a_{n=2, \ldots, N / 2+1}$ with the amplitude $T_{n=1, \ldots, N / 2}$ before the interface site $a_{N / 2+1}$ and possesses the the long-range hopping between the last site $a_{N+1}$ and all other $a$-type sites $a_{n=N / 2+1, \ldots, N}$ with the amplitude $T_{n=N / 2+1, \ldots, N}$ after the interface site $a_{N / 2+1}$.

where $J_{1}=J+\cos \theta\left(J_{2}=J-\cos \theta\right)$ is still the modulated NN hopping amplitude and $T_{1}=T_{2}=\ldots=T_{N}=T J_{2}$ is the long-range hopping amplitude.

According to the conclusions obtained in Sec. II A and Sec. II B, it is easy to infer that the interface induces a topological channel to implement the state transfer between the initial states $|\Psi\rangle_{i, 2}=$ $|0,0,0,0, \ldots, 0,1,0, \ldots, 0,0,0,0\rangle$ and the ideal final state $\quad|\Psi\rangle_{f, 2}=\frac{1}{\sqrt{N+2}}|1,1,0,1, \ldots, 1,0,1, \ldots, 1,0,1,1\rangle$ when $T=1$ or implement the state transfer between the initial states $|\Psi\rangle_{i, 2}$ and the ideal final state $|\Psi\rangle_{f, 2}^{\prime}=$ $\frac{1}{\sqrt{N}}|0,1,0,1, \ldots, 1,0,1, \ldots, 1,0,1,0\rangle$ when $T \gg 1$. To further verify the above inference, we simulate the fidelity of the two kinds of state transfer process versus the ramping speed $\Omega$ and parameter $T$, as shown in Figs. 6(a) and 6(b). The numerical results clearly reveal that we can always find
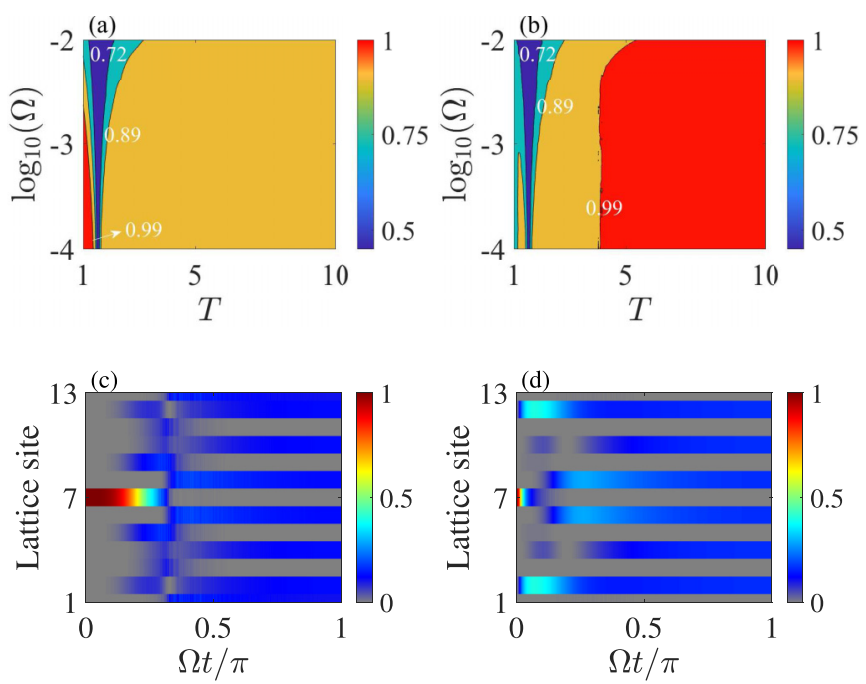

FIG. 6. Fidelity and evolution process of the initial state $|\Psi\rangle_{i, 2}$. (a) The fidelity between the evolved final state and the ideal final state $|\Psi\rangle_{f, 2}$ versus the ramping speed $\Omega$ and parameter $T$. (b) The fidelity between the evolved final state and the ideal final state $|\Psi\rangle_{f, 2}^{\prime}$ versus the ramping speed $\Omega$ and parameter $T$. (c) The process of the evolution for the initial state $|\Psi\rangle_{i, 2}$ when parameters satisfy $\Omega=$ $10^{-5} \mathrm{~J}$ and $T=1.1 \mathrm{~J}$. (d) The process of the evolution for the initial state $|\Psi\rangle_{i, 2}$ when parameters satisfy $\Omega=10^{-5} \mathrm{~J}$ and $T=8 \mathrm{~J}$. The other parameter takes $L=2 N+1$ with $N=6$. The unit is $J=1$. an appropriate $\Omega$ to implement the first (second) kind of state transfer with a high enough fidelity when the parameter $T$ approximately satisfies $T<1.5 \mathrm{~J}(T>1.5 \mathrm{~J})$. The two kinds of state transfer processes are shown in Figs. 6(c) and 6(d). We stress that the two kinds of state transfers induced by the interface also correspond to two topological routers, in which the particle initially prepared at the interface site can be distributed toward multiple output ports. Compared with the topological routers mentioned in Sec. II A and Sec. II B, the present topological router induced by the interface can realize the symmetric (the interface site is the symmetry center) distributions of the initial particle.

\section{EXPERIMENT AND DISCUSSION}

The different kinds of topological routers have many potential applications in quantum information processing, i.e., taking the topological routers as the topological channel to implement the large-scale entanglement distribution. Thus, before concluding, we give a brief discussion with respect to the experimental realization of the topological routers.

\section{A. Realization of the long-range hopping in the Su-Schrieffer-Heeger chain}

The cold atoms trapped in an optical lattice [50-54] are widely used to investigate various topological issues, including the topological boundary state [55-58], topological phase transition [59-61], and topological invariant [59,62-64]. Note that the controllable NNN hopping between two adjacent unit cells can be realized based on parametrically coupled atomic momentum states in the cold-atom system [65]. Moreover, the multifold long-range hopping terms between one certain site and other sites can also be realized via the shallow optical lattice $[66,67]$, in which the long-range hopping amplitudes decay exponentially with distance between two sites. Although various kinds of long-range hopping can be constructed in cold-atom systems, the given long-range hopping form (the long-range hopping only added between the first $a$-type site and other $a$-type sites with the identical hopping amplitudes) in this paper seems to still have great obstacles in cold-atom systems.

In addition to the cold-atom system, the superconducting circuit lattice [68-70] consisting of superconducting qubits and superconducting resonators has also attracted much more 
attention for the mapping of topological matter due to the designability of geometric pattern and coupling configuration in space. Depending on the flexible designability of the superconducting circuit lattice, the long-range hopping between two sites can be designed individually, such as the long-range hopping shown in Fig. 1. What is more important is that the strength of the long-range hopping terms can be tuned individually and adiabatically via the external control [69], including the superconducting quantum interference devices, the flux-bias line, and the additional coupler circuits. Experimentally, the typical coupling between two superconducting resonators can be tuned in the range of $0 \sim 100 \mathrm{MHz}$ [69], together with the coupling between the superconducting resonator and qubit in the range of $0 \sim 400 \mathrm{MHz}$, providing a considerably wide tuning range for the amplitudes of the $\mathrm{NN}$ and long-range hopping. In this way, the superconducting circuit lattice based topological router can be implemented as the topological channel to realize the large-scale distribution.

\section{B. Analysis of the adiabatic evolution process for the topological router}

Note that, as shown in Sec. II, the various kinds of topological routers need to satisfy the strict adiabatic evolution demand to avoid the evolution process to integrate into the bulk, indicating that the ramping speed $\Omega$ should be small enough to ensure that the whole evolution process is along the gap state and passes the point around the minimal energy space $\Delta_{E}$ safely. However, the small enough ramping speed $\Omega$ usually means that the periodic parameter should be varied extremely slowly, corresponding to an extremely long evolution time, which brings great challenges for the practical experimental platform. For example, in the superconducting circuit lattice mentioned above, the extremely slow varied parameter means that the external control field of the superconducting circuit lattice should be modulated adiabatically, which may cause certain challenges for the current experimental techniques. At the same time, the extremely long evolution time also may be beyond the life of the superconducting qubits, which inevitably leads the decoherence effect, leading the topological router to be invalid in superconducting circuit experiments.

To avoid these obstacles, one alternative method is that we enlarge the ramping speed $\Omega$ directly. Generally, the order of magnitude for the ramping speed $\Omega$ can be roughly estimated by the formula of $\sqrt{\Omega}<\Delta_{E}$ [45], meaning that the restriction of the ramping speed $\Omega$ can be relaxed appropriately via the larger minimal energy space $\Delta_{E}$. Meanwhile, the finite-size effect of the $\mathrm{SSH}$ model determines that the energy gap is closely related to the size of the system, in which the energy gap exhibits an exponential decay behavior with the increasing of the size of the chain, meaning that we may enlarge the minimal energy space $\Delta_{E}$ to obtain a relatively larger $\Omega$ via decreasing the size of the SSH chain appropriately. In Fig. 7(a), we plot the minimal energy space $\Delta_{E}$ versus the size of the chain $L$ when the long-range hopping satisfies $T_{1}=T_{2}=\ldots=T_{N}=J_{2}$ (the topological router shown in Sec. II A). The numerical results reveal that the minimal energy space $\Delta_{E}$ indeed exhibits an exponential decay behavior approximately with the increasing of the size of the

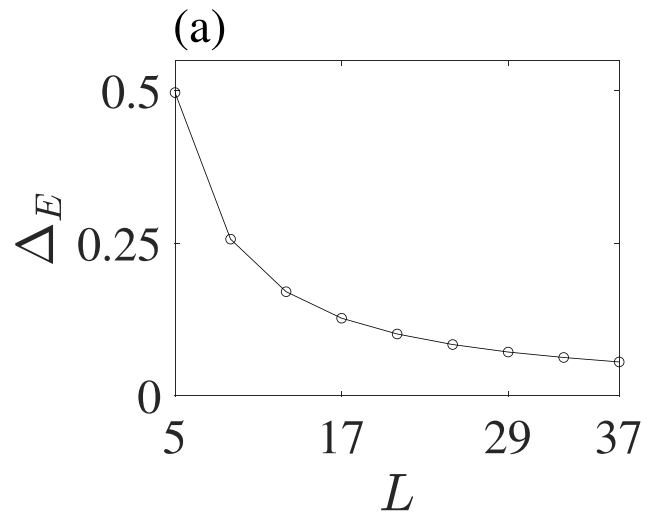

(b)

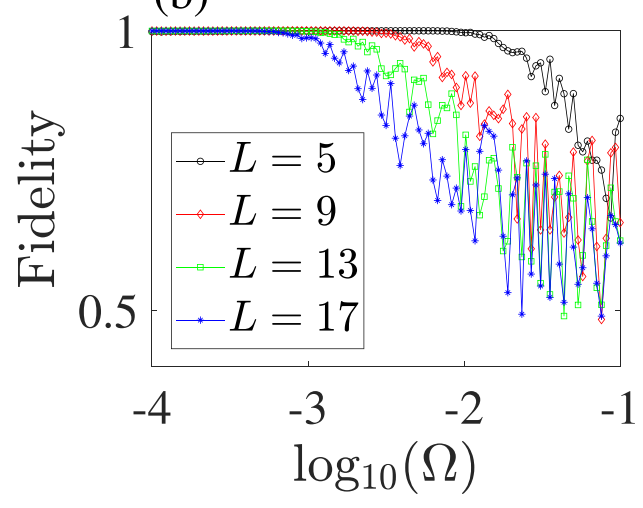

FIG. 7. (a) The minimal energy space $\Delta_{E}$ versus the size of the lattice $L$ when $T_{1}=T_{2}=\ldots=T_{N}=J_{2}$. (b) The fidelity of the topological router versus the ramping speed $\Omega$ corresponding to different size of the lattice. The unit is $J=1$.

chain. Especially, when the size of the chain is taken $L=5$, the minimal energy space $\Delta_{E}$ can even reach about $0.5 \mathrm{~J}$. To further estimate the effects of the increasing size of the lattice on the ramping speed $\Omega$, we plot the fidelity of the topological router shown in Sec. II A versus the ramping speed $\Omega$ when the chain takes different sizes, as shown in Fig. 7(b). Obviously, the shorter chain corresponds a wider range of the ramping speed $\Omega$ with fidelity being close to 1 . For the small SSH chain with the size of $L=5$, we find that the fidelity can still be equal to 1 even when $\Omega=10^{-2} J$. In superconducting circuit lattice experiments, the current state of the art for superconducting circuits can produce a medium-sized superconducting quantum computer with qubit numbers of 50-100 [45], which means that the lattice with the size of $L=5$ can be easily implemented. Besides, in the superconducting circuit lattice, the ramping speed $\Omega=0.01 J$ with $J / 2 \pi=250 \mathrm{MHz}$ corresponds to the time of the transfer process $t_{\text {total }}=\pi / \Omega=$ $0.2 \mu \mathrm{s}$, which is much shorter than typical qubit decoherence time 30-140 $\mu \mathrm{s}$ [71]. Actually, even for the longer chain with $L=13$, the ramping speed $\Omega=0.001$ still ensures that the time of the transfer process $t_{\text {total }}=2 \mu \mathrm{s}$ is much shorter than the qubit decoherence times. Thus, increasing the ramping speed $\Omega$ via decreasing the size of the chain is a totally feasible way in the experiment.

Besides the way to decrease the size of the chain directly, we also may use the shortcut method to accelerate the trans- 
fer process; e.g., we can apply the shortcut-to-adiabaticity method to pass the point around the minimal energy space and realize the adiabatic quantum state transfer [45]. Note that, in Ref. [46], the author induces a topological interface state to realize the accelerated quantum state transfer between the left and right edge states. Via designing the adiabatic passage in the dressed three-level description of the left, right, and interface states, the fast state transfer between the left and right states can be implemented. Since the topological router mentioned in Sec. II C also has a topological interface, the adiabatic passage method may provide enlightening suggestions for our topological router. Moreover, in Ref. [47], the authors show that the fast and robust quantum state transfer between two edge states can be realized via introducing the NNN hopping between $a$-type sites. This method actually maps the chain into a three-level space via introducing the hopping between NNN $a$-type sites. In this way, via designing the hopping rate in the three-level space using the shortcut technique, the state transfer between the edge sites can be realized in a fast and robust way. Differently, the SSH model with the long-range hopping mentioned in Fig. 1 cannot be mapped into the similar three-level space since the long-range hopping terms are totally different. However, we may map the present model into a multilevel space, by which we may realize the acceleration of the state transfer via designing the coupling using the multilevel shortcut technique. At the same time, we must clarify that the topological router may not realize the splitting at each output port with the equal probability after designing the coupling using the multilevel shortcut technique, which is against our original intention.

\section{CONCLUSIONS}

In conclusion, we have proposed several different parameter regimes to induce the topological router based on the SSH model with the NN hopping modulations. We demonstrate that, when the introduced long-range hopping amplitudes between the first site $a_{1}$ and other $a$-type sites are equal to the intercell hopping strength, the long-range hopping opens up a special topological channel. By dint of this topological channel, the particle initially prepared at the last site can be simultaneously transferred into the first site and all of the even sites with the approximately equal probabilities, implying the potential probability to implement the split-flow process with multiple output ports, such as the topological router. We show that, due to the topological protection originating from the energy gap, the present topological router is spontaneously immune to the mild disorder added into the on-site energy and NN hopping. Furthermore, depending on the designability of the long-range hopping terms, we reveal that the amounts of the output ports for the topological router can be modulated via reducing the corresponding long-range hopping terms. Dramatically, we find that the system experiences a phase transition with the increasing of the long-range hopping amplitudes, in which the new topological router with output ports only at all even sites can be implemented via the gap state. Furthermore, we also explore the effects of the topological interface on the topological routers, and show that the topological routers can own the output ports in a symmetric way. We stress that the topological routers are expected to be implemented in the superconducting circuit system and greatly expand the applications of topological matter in quantum information processing.

\section{ACKNOWLEDGMENTS}

This work was supported by the National Natural Science Foundation of China under Grants No. 61822114, No. 12074330, No. 62071412, No. 11874132, and No. 61575055.

\section{APPENDIX}

The number of the output ports for the topological router mentioned in Sec. II A can be tuned via designing the longrange hopping between $a$-type sites. For example, when the long-range hopping amplitudes between $a$-type sites satisfy $T_{1}=T_{2}=\ldots=T_{N-1}=J_{2}$, it is easy to find that the energy spectrum owns a gap state, in which it is mainly localized at the last site within $\theta \in[0,0.5 \pi]$ while is mainly localized at sites $a_{1}, b_{1}, b_{2}, \ldots, b_{N-1}$ within $\theta \in[0.5 \pi, \pi]$, as shown in Figs. 8(a) and 8(b). This special distribution of the gap state indicates that the gap state can be treated as the topological channel to implement the state transfer between the states of $|0,0,0,0, \ldots, 0,0,0,1\rangle$ and $\frac{1}{\sqrt{N}}|1,1,0,1, \ldots, 1,0,0,0\rangle$. Compared with the case shown in Fig. 2(b), the present topological channel obviously reduces one output port. Similarly, we can further decrease the number of the output
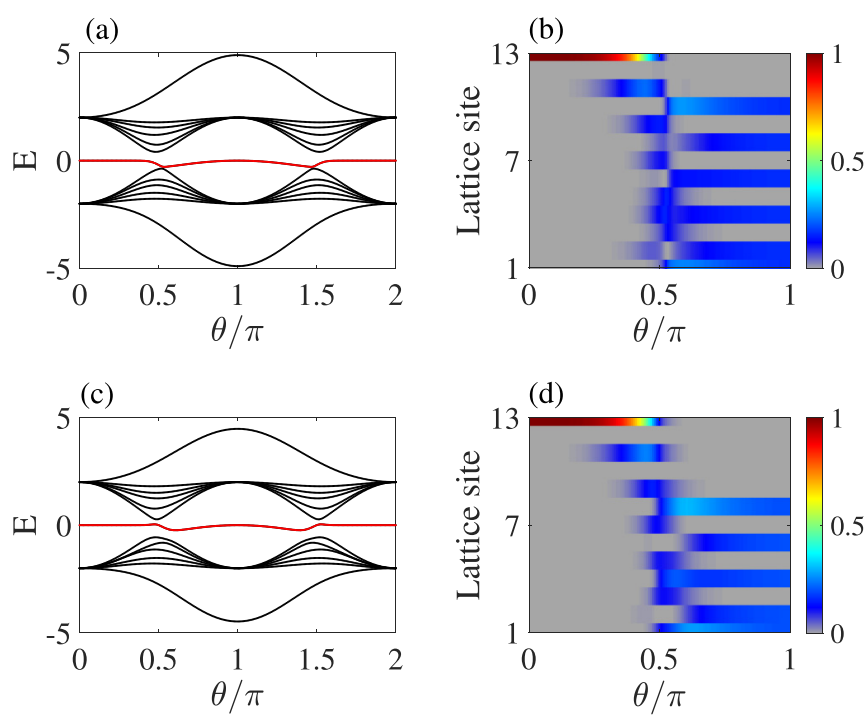

FIG. 8. Energy spectrum and distribution of gap state. (a) The energy spectrum of the SSH chain when $T_{1}=T_{2}=\ldots=T_{N-1}=J_{2}$. The energy spectrum has a gap state (red line). (b) The distribution of the gap state in (a) versus the periodic parameter $\theta$, in which the gap state is mainly localized at the last site within $\theta \in[0,0.5 \pi]$ while it is mainly localized at sites $a_{1}$ and $b_{n=1, \ldots, N-1}$ uniformly within $\theta \in[0.5 \pi, \pi]$. (c) The energy spectrum of the SSH chain when $T_{1}=T_{2}=\ldots=T_{N-2}=J_{2}$. The energy spectrum has a gap state (red line). (d) The distribution of the gap state in (c) versus the periodic parameter $\theta$, in which the gap state is mainly localized at the last site within $\theta \in[0,0.5 \pi]$ while it is mainly localized at sites $a_{1}$ and $b_{n=1, \ldots, N-2}$ uniformly within $\theta \in[0.5 \pi, \pi]$. The other parameter takes $L=2 N+1$ with $N=6$. The unit is $J=1$. 
ports via continuously reducing the long-range hopping terms, such as $T_{1}=T_{2}=\ldots=T_{N-2}=J_{2}$. The relevant spectrum and the distribution of the gap state are shown in Figs. 8(c) and $8(\mathrm{~d})$. The numerical results clearly show that the state transfer between the states of $|0,0,0,0, \ldots, 0,0,0,1\rangle$ and

[1] M. Saffman, T. G. Walker, and K. Mølmer, Quantum information with Rydberg atoms, Rev. Mod. Phys. 82, 2313 (2010).

[2] D. Suter and G. A. Álvarez, Colloquium: Protecting quantum information against environmental noise, Rev. Mod. Phys. 88, 041001 (2016).

[3] A. Galindo and M. A. Martín-Delgado, Information and computation: Classical and quantum aspects, Rev. Mod. Phys. 74, 347 (2002).

[4] L. M. Duan and C. Monroe, Colloquium: Quantum networks with trapped ions, Rev. Mod. Phys. 82, 1209 (2010).

[5] E. Biolatti, R. C. Iotti, P. Zanardi, and F. Rossi, Quantum Information Processing with Semiconductor Macroatoms, Phys. Rev. Lett. 85, 5647 (2000).

[6] M. Bourennane, M. Eibl, S. Gaertner, C. Kurtsiefer, A. Cabello, and $\mathrm{H}$. Weinfurter, Decoherence-Free Quantum Information Processing with Four-Photon Entangled States, Phys. Rev. Lett. 92, 107901 (2004).

[7] D. Ahn, J. Lee, M. S. Kim, and S. W. Hwang, Selfconsistent non-Markovian theory of a quantum-state evolution for quantum-information processing, Phys. Rev. A 66, 012302 (2002).

[8] C. Z. Peng, J. Zhang, D. Yang, W. B. Gao, H. X. Ma, H. Yin, H. P. Zeng, T. Yang, X.-B. Wang, and J.-W. Pan, Experimental Long-Distance Decoy-State Quantum Key Distribution Based on Polarization Encoding, Phys. Rev. Lett. 98, 010505 (2007).

[9] B. Vlastakis, G. Kirchmair, Z. Leghtas, S. E. Nigg, L. Frunzio, S. M. Girvin, M. Mirrahimi, M. H. Devoret, and R. J. Schoelkopf, Deterministically encoding quantum information using 100-photon Schrödinger cat states, Science 342, 607 (2013).

[10] P. Zanardi and F. Rossi, Quantum Information in Semiconductors: Noiseless Encoding in a Quantum-Dot Array, Phys. Rev. Lett. 81, 4752 (1998).

[11] M. Bourennane, A. Karlsson, and G. Björk, Quantum key distribution using multilevel encoding, Phys. Rev. A 64, 012306 (2001).

[12] J. I. Cirac, P. Zoller, H. J. Kimble, and H. Mabuchi, Quantum State Transfer and Entanglement Distribution among Distant Nodes in a Quantum Network, Phys. Rev. Lett. 78, 3221 (1997).

[13] D. N. Matsukevich and A. Kuzmich, Quantum state transfer between matter and light, Science 306, 663 (2004).

[14] M. Christandl, N. Datta, A. Ekert, and A. J. Landahl, Perfect State Transfer in Quantum Spin Networks, Phys. Rev. Lett. 92, 187902 (2004).

[15] Y. D. Wang and A. A. Clerk, Using Interference for High Fidelity Quantum State Transfer in Optomechanics, Phys. Rev. Lett. 108, 153603 (2012).

[16] J. Zhang, K. Peng, and S. L. Braunstein, Quantum-state transfer from light to macroscopic oscillators, Phys. Rev. A 68, 013808 (2003).

[17] M. Ouyang and D. D. Awschalom, Coherent spin transfer between molecularly bridged quantum dots, Science 301, 1074 (2003).
$\frac{1}{\sqrt{N-1}}|1,1,0,1, \ldots, 0,0,0,0\rangle$ can be realized via the adiabatic pumping of the gap state. In this way, we can tune the number of the output ports for the topological router, which greatly improves the usability of the topological router in practical applications.

[18] T. Brandes and T. Vorrath, Adiabatic transfer of electrons in coupled quantum dots, Phys. Rev. B 66, 075341 (2002).

[19] Y. He, Y. M. He, Y. J. Wei, X. Jiang, K. Chen, C. Y. Lu, J. W. Pan, C. Schneider, M. Kamp, and S. Höfling, Quantum State Transfer from a Single Photon to a Distant Quantum-Dot Electron Spin, Phys. Rev. Lett. 119, 060501 (2017).

[20] Y. Masumoto, H. Takagi, H. Umino, and E. Suzumura, Fast electron transfer from $\mathrm{PbSe}$ quantum dots to $\mathrm{TiO}_{2}$, Appl. Phys. Lett. 100, 252106 (2012).

[21] S. B. Zheng and G. C. Guo, Efficient Scheme for Two-Atom Entanglement and Quantum Information Processing in Cavity QED, Phys. Rev. Lett. 85, 2392 (2000).

[22] C. P. Yang, S. I. Chu, and S. Han, Possible realization of entanglement, logical gates, and quantum-information transfer with superconducting-quantum-interference-device qubits in cavity QED, Phys. Rev. A 67, 042311 (2003).

[23] C. P. Yang, S. I. Chu, and S. Han, Quantum Information Transfer and Entanglement with Squid Qubits in Cavity QED: A Dark-State Scheme with Tolerance for Nonuniform Device Parameter, Phys. Rev. Lett. 92, 117902 (2004).

[24] M. A. Sillanpää, J. I. Park, and R. W. Simmonds, Coherent quantum state storage and transfer between two phase qubits via a resonant cavity, Nature (London) 449, 438 (2007).

[25] W. L. Yang, Z. Q. Yin, Z. Y. Xu, M. Feng, and C. H. Oh, Quantum dynamics and quantum state transfer between separated nitrogen-vacancy centers embedded in photonic crystal cavities, Phys. Rev. A 84, 043849 (2011).

[26] Q. Chen, W. L. Yang, and M. Feng, Controllable quantum state transfer and entanglement generation between distant nitrogen-vacancy-center ensembles coupled to superconducting flux qubits, Phys. Rev. A 86, 022327 (2012).

[27] Z. B. Feng, Robust quantum state transfer between a Cooperpair box and diamond nitrogen-vacancy centers, Phys. Rev. A 91, 032307 (2015)

[28] M. Z. Hasan and C. L. Kane, Colloquium: Topological insulators, Rev. Mod. Phys. 82, 3045 (2010).

[29] X. L. Qi and S. C. Zhang, Topological insulators and superconductors, Rev. Mod. Phys. 83, 1057 (2011).

[30] C. K. Chiu, J. C. Teo, A. P. Schnyder, and S. Ryu, Classification of topological quantum matter with symmetries, Rev. Mod. Phys. 88, 035005 (2016).

[31] A. Bansil, H. Lin, and T. Das, Colloquium: Topological band theory, Rev. Mod. Phys. 88, 021004 (2016).

[32] V. M. Martinez Alvarez, J. E. Barrios Vargas, and L. E. F. Foa Torres, Non-Hermitian robust edge states in one dimension: Anomalous localization and eigenspace condensation at exceptional points, Phys. Rev. B 97, 121401(R) (2018).

[33] Q. Wu, L. Du, and V. E. Sacksteder, Robust topological insulator conduction under strong boundary disorder, Phys. Rev. B 88, 045429 (2013)

[34] O. Gröning, S. Wang, X. Yao, C. A. Pignedoli, G. Borin Barin, C. Daniels, A. Cupo, V. Meunier, X. Feng, A. Narita, K. Müllen, P. Ruffieux, and R. Fasel, Engineering of robust topological 
quantum phases in graphene nanoribbons, Nature (London) 560, 209 (2018).

[35] L. Chen, Z. F. Wang, and F. Liu, Robustness of two-dimensional topological insulator states in bilayer bismuth against strain and electrical field, Phys. Rev. B 87, 235420 (2013).

[36] T. Paananen and T. Dahm, Magnetically robust topological edge states and flat bands, Phys. Rev. B 87, 195447 (2013).

[37] N. Lang and H. P. Büchler, Topological networks for quantum communication between distant qubits, npj Quantum Inf. 3, 47 (2017).

[38] S. Longhi, G. L. Giorgi, and R. Zambrini, Landau-Zener topological quantum state transfer, Adv. Quantum Technol. 2, 1800090 (2019).

[39] L. Qi, G. L. Wang, S. Liu, S. Zhang, and H. F. Wang, Controllable photonic and phononic topological state transfers in a small optomechanical lattice, Opt. Lett. 45, 2018 (2020).

[40] L. N. Zheng, L. Qi, L. Y. Cheng, H. F. Wang, and S. Zhang, Defect-induced controllable quantum state transfer via a topologically protected channel in a flux qubit chain, Phys. Rev. A 102, 012606 (2020).

[41] L. Qi, Y. Xing, X. D. Zhao, S. Liu, X. Han, W. X. Cui, S. Zhang, and H. F. Wang, Tunable topological beam splitter in superconducting circuit lattice, Quantum Rep. 3, 1 (2021).

[42] Y. E. Kraus, Y. Lahini, Z. Ringel, M. Verbin, and O. Zilberberg, Topological States and Adiabatic Pumping in Quasicrystals, Phys. Rev. Lett. 109, 106402 (2012).

[43] M. Verbin, O. Zilberberg, Y. Lahini, Y. E. Kraus, and Y. Silberberg, Topological pumping over a photonic Fibonacci quasicrystal, Phys. Rev. B 91, 064201 (2015).

[44] C. Dlaska, B. Vermersch, and P. Zoller, Robust quantum state transfer via topologically protected edge channels in dipolar arrays, Quantum Sci. Technol. 2, 015001 (2017).

[45] F. Mei, G. Chen, L. Tian, S. L. Zhu, and S. Jia, Robust quantum state transfer via topological edge states in superconducting qubit chains, Phys. Rev. A 98, 012331 (2018).

[46] S. Longhi, Topological pumping of edge states via adiabatic passage, Phys. Rev. B 99, 155150 (2019).

[47] F. M. D’Angelis, F. A. Pinheiro, D. Guéry-Odelin, S. Longhi, and F. M. C. Impens, Fast and robust quantum state transfer in a topological Su-Schrieffer-Heeger chain with next-to-nearestneighbor interactions, Phys. Rev. Res. 2, 033475 (2020).

[48] L. Qi, G. L. Wang, S. Liu, S. Zhang, and H. F. Wang, Engineering the topological state transfer and topological beam splitter in an even-sized Su-Schrieffer-Heeger chain, Phys. Rev. A 102, 022404 (2020).

[49] J. K. Asbóth, L. Oroszlány, and A. Pályi, A Short Course on Topological Insulators, Lecture Notes in Physics Vol. 919 (Springer, Cham, 2016), pp. 19-20.

[50] N. R. Cooper, J. Dalibard, and I. B. Spielman, Topological bands for ultracold atoms, Rev. Mod. Phys. 91, 015005 (2019).

[51] J. Dalibard, F. Gerbier, G. Juzeliūnas, and P. Öhberg, Colloquium: Artificial gauge potentials for neutral atoms, Rev. Mod. Phys. 83, 1523 (2011).

[52] N. Goldman, J. C. Budich, and P. Zoller, Topological quantum matter with ultracold gases in optical lattices, Nat. Phys. 12, 639 (2016).

[53] F. Mei, S. L. Zhu, Z. M. Zhang, C. H. Oh, and N. Goldman, Simulating $Z_{2}$ topological insulators with cold atoms in a onedimensional optical lattice, Phys. Rev. A 85, 013638 (2012).
[54] D. W. Zhang, Y. Q. Zhu, Y. X. Zhao, H. Yan, and S. L. Zhu, Topological quantum matter with cold atoms, Adv. Phys. 67, 253 (2018)

[55] L. J. Lang, X. Cai, and S. Chen, Edge States and Topological Phases in One-Dimensional Optical Superlattices, Phys. Rev. Lett. 108, 220401 (2012).

[56] T. D. Stanescu, V. Galitski, and S. Das Sarma, Topological states in two-dimensional optical lattices, Phys. Rev. A 82, 013608 (2010).

[57] N. Goldman, J. Beugnon, and F. Gerbier, Detecting Chiral Edge States in the Hofstadter Optical Lattice, Phys. Rev. Lett. 108, 255303 (2012).

[58] X. J. Liu, Z. X. Liu, and M. Cheng, Manipulating Topological Edge Spins in a One-Dimensional Optical Lattice, Phys. Rev. Lett. 110, 076401 (2013).

[59] Z. Xu, Y. Zhang, and S. Chen, Topological phase transition and charge pumping in a one-dimensional periodically driven optical lattice, Phys. Rev. A 96, 013606 (2017).

[60] G. Liu, N. Hao, S. L. Zhu, and W. M. Liu, Topological superfluid transition induced by a periodically driven optical lattice, Phys. Rev. A 86, 013639 (2012).

[61] A. R. Kolovsky, Topological phase transitions in tilted optical lattices, Phys. Rev. A 98, 013603 (2018).

[62] D. W. Zhang, Y. X. Zhao, R. B. Liu, Z. Y. Xue, S. L. Zhu, and Z. D. Wang, Quantum simulation of exotic $\mathcal{P} \mathcal{T}$-invariant topological nodal loop bands with ultracold atoms in an optical lattice, Phys. Rev. A 93, 043617 (2016).

[63] S. T. Wang, D. L. Deng, and L. M. Duan, Probe of Three-Dimensional Chiral Topological Insulators in an Optical Lattice, Phys. Rev. Lett. 113, 033002 (2014).

[64] F. N. Ünal, B. Seradjeh, and A. Eckardt, How to Directly Measure Floquet Topological Invariants in Optical Lattices, Phys. Rev. Lett. 122, 253601 (2019).

[65] F. A. An, E. J. Meier, and B. Gadway, Engineering a FluxDependent Mobility Edge in Disordered Zigzag Chains, Phys. Rev. X 8, 031045 (2018).

[66] J. Biddle, D. J. Priour, B. Wang, and S. Das Sarma, Localization in one-dimensional lattices with non-nearest-neighbor hopping: Generalized Anderson and Aubry-André models, Phys. Rev. B 83, 075105 (2011).

[67] J. Biddle and S. Das Sarma, Predicted Mobility Edges in One-Dimensional Incommensurate Optical Lattices: An Exactly Solvable Model of Anderson Localization, Phys. Rev. Lett. 104, 070601 (2010).

[68] D. I. Tsomokos, S. Ashhab, and F. Nori, Using superconducting qubit circuits to engineer exotic lattice systems, Phys. Rev. A 82, 052311 (2010).

[69] S. Schmidt and J. Koch, Circuit QED lattices: Towards quantum simulation with superconducting circuits, Ann. Phys. 525, 395 (2013).

[70] D. Marcos, P. Rabl, E. Rico, and P. Zoller, Superconducting Circuits for Quantum Simulation of Dynamical Gauge Fields, Phys. Rev. Lett. 111, 110504 (2013).

[71] R. Barends, J. Kelly, A. Megrant, D. Sank, E. Jeffrey, Y. Chen, Y. Yin, B. Chiaro, J. Mutus, C. Neill, P. O’Malley, P. Roushan, J. Wenner, T. C. White, A. N. Cleland, and John M. Martinis, Coherent Josephson Qubit Suitable for Scalable Quantum Integrated Circuits, Phys. Rev. Lett. 111, 080502 (2013). 